\title{
Consecuencias sobre el empleo por el impacto del COVID-19, en el entorno de los negocios de la Región 6
}

Consequences on employment due to the impact of COVID-19, in the business environment of Region 6

Edison Becerra Molina. ${ }^{1}$, Gina Cuadrado Sánchez. ${ }^{2} \&$ Pedro Astudillo Arias. ${ }^{3}$ Recibido: 07-08-2021 / Revisado: 19-08-2021 /Aceptado: 10-09-2021/ Publicado: 05-10-2021

\begin{abstract}
DOI: https://doi.org/10.33262/concienciadigital.v4i4.1905

Introduction. The work environment is the activity that people carry out either as a duty or an activity dependent on the profession to cover their needs and desires, as the result of the interaction of motivational factors and salary. Target. Analyze the effects on employment in times of pandemic in MSMEs through an analysis of the strategies adopted by employers and the measures taken by the government. Methodology. The research design started from the positivist research paradigm, non-experimental and transectional field design with documentary support, descriptive research level, the population was 77,289 and the sample 471, the questionnaire instrument was used, it was validated by trial of experts with experience in the labor area and methodology, qualifying validity, clarity, coherence, and relevance. Results. The reasons for dismissing workers due to the presence of COVID-19 were $40.55 \%$ did not have to resort to this measure, however, the main causes for which personnel were dismissed $23.57 \%$ opted for untimely dismissal, $12.53 \%$ resigned, $11.04 \%$ termination of employment contract, $5.52 \%$ termination of the job, $4.03 \%$ due to company liquidation, $2.76 \%$ took retirement. It was possible to show that there is a high percentage of people who ceased their functions due

\footnotetext{
1 Universidad Católica de Cuenca, Carrera de Contabilidad y Auditoría, provincia Azuay, código, jbecerram@ucacue.edu.ec, Orcid.Org ID: 0000-0002-6397-9493

${ }_{2}^{2}$ Universidad Católica de Cuenca, Carrera de Contabilidad y Auditoría, provincia Azuay, código, gcuadrado@ucacue.edu.ec, Orcid.Org ID: 0000-0002-4259-4906

${ }^{3}$ Universidad Católica de Cuenca, Carrera de Contabilidad y Auditoría, provincia Azuay, código, pastudillo@ucacue.edu.ec, Orcid.Org ID:0000-0003-3088-8322
} 
to the suppression of their activities, these measures were assumed by the business owners due to the temporary nature of the pandemic that resulted in large losses and casualties in the sale of its products and services, due to the temporary suspension of its activities, which led to countless layoffs and closure of companies. Conclution. The negative effects on the economic aspect that the pandemic brings, show that employment has been affected, by the increase in unemployment and underemployment, by the closure and reduction of wages and less access to social protection, however, the prompt The government's response mitigated this panorama through a series of public policies.

Keywords: COVID-19, Economy, MSMEs, Unemployment, Labor restrictions, Regulatory incentives.

\section{Resumen}

Introducción. El ámbito laboral es la actividad que las personas realizan ya sea como deber o actividad dependiente de la profesión para cubrir sus necesidades y deseos, como el resultado de la interacción de factores de motivación y sueldo. Objetivo. Analizar los efectos sobre el empleo en época de pandemia en las Mipymes a través de un análisis de las estrategias adoptadas por los empleadores y las medidas tomadas por el gobierno. Metodología. El diseño de la investigación partió del paradigma de investigación positivista, diseño de campo no experimental y transeccional con apoyo documental, nivel de investigación descriptiva, la población fue 77.289 y la muestra 471, se utilizó el instrumento del cuestionario, el mismo fue validado mediante juicio de expertos con experiencia en el área laboral y metodología, calificando validez, claridad, coherencia, y relevancia. Resultados. Las razones de despido de trabajadores por la presencia del COVID-19, fueron $40.55 \%$ no tuvo que acudir a esta medida, sin embargo, las principales causas por las que se despidió personal $23.57 \%$ optó por el despido intempestivo, $12.53 \%$ renunció, $11.04 \%$ terminación de contrato de trabajo, $5.52 \%$ supresión del puesto de trabajo, $4.03 \%$ a causa de liquidación de la empresa, $2.76 \%$ se acogieron a la jubilación. Fue posible evidenciar que existe un alto porcentaje de personas que cesaron de sus funciones a causa de la supresión de sus actividades, estas medidas fueron asumidas por los dueños de los negocios a causa de la temporalidad de pandemia que resultó en grandes pérdidas y bajas en la venta de sus productos y servicios, por la suspensión temporal de sus actividades, lo cual desencadeno en un sinfín de despidos y cierre de las empresas. Conclusión. Los efectos negativos en el aspecto económico que acarrea la pandemia, exponen que el empleo se ha visto afectado, por el aumento del desempleo y el subempleo, por el cierre y reducción de salarios y menor acceso a la protección social, sin embargo, la pronta respuesta del gobierno atenúo este panorama mediante una serie de políticas públicas.

Palabras claves: COVID-19, Economía, Mipymes, Desempleo, Restricciones laborales, Incentivos normativos. 


\section{Introducción}

La pandemia actual está teniendo un gran impacto económico, social y un cambio a escala mundial para los trabajadores en todos los sectores de la economía y en todo tipo de negocios. La oferta de bienes y servicios han sido afectados y por ende la demanda se ha reducido en forma notoria. Esta situación ha ocasionado que la economía de muchas empresas se deteriore y a su vez, volcando la mirada de las empresas a apreciar el valor estratégico que tienen las personas en todas sus operaciones, además desafió la adaptabilidad de los ordenamientos jurídicos en áreas especialmente sensibles a los cambios económicos como lo es el ámbito del trabajo.

La realidad del impacto es notorio con la caída económica de las micro, pequeñas y medianas empresas (Mipymes) las cuales son propulsoras de empleo y producción en la sociedad, llevándolas a cierre de estos negocios, interrumpiendo bruscamente las cadenas de producción dejando en evidencia la debilidad de los modelos operativos y las cadenas de suministros de las empresas y con ello la inactividad económica, ocasionando la suspensión del flujo de ingresos.

La misión de la presente investigación es analizar la situación en el ámbito laboral de la mayoría de las micro, pequeñas y medianas de la región zonal 6 del Ecuador durante la pandemia del COVID 19, proporcionando elementos de análisis que propenden a fortalecer la sustentabilidad de los negocios con la finalidad de contribuir a la solución de los problemas en el ámbito laboral.

De acuerdo con lo antes referido, se realiza un análisis basado en los estudios recabados relacionados con el impacto de la COVID-19, en el desarrollo económico de las Mipymes de la región zonal 6. El procesamiento de la información se realizó a través de la técnica de análisis de contenidos. Este procedimiento, se ejecutó para corroborar la evolución de la COVID-19 y sus principales impactos en el desarrollo económico, centrando la atención en el tema laboral.

En aspectos generales, se prevé un aumento del desempleo mundial que oscila entre 5,3 millones (caso "más favorable") y 24,7 millones de personas (caso "más desfavorable"), con respecto a un valor de referencia de 188 millones de desempleados en 2019 (OIT, 2020). Cabe destacar que el Ecuador ha ido cursando ciertas circunstancias que han involucrado acontecimientos enmarcados en el plano financiero económico externo, esto denota un desequilibrio directo para los ingresos empresariales, que, en lugar de subsanar la brecha laboral, únicamente basan sus esfuerzos en la subsistencia.

La pandemia ocasionada por el COVID-19 ha puesto en evidencia el sempiterno declive de las leyes caducas en materia de la subsistencia de las plazas de trabajo, no obstante, y obedeciendo a la estadística laboral que muestra valores reales, cada punto porcentual que logra sustraerse de las cifras del desempleo y adherirse al siempre empleo digno, se revierte en ingresos para los hogares y contribuye con el producto Interno Bruto- PIB; tornándose en una inyección directa a la tranquilidad y buen vivir. 
Este trabajo es parte del proyecto de investigación denominado "La importancia de la información contable y financiera ante la incertidumbre del COVID-19 en las Mipymes en el entorno de los negocios en la región 6", el mismo que se justifica porque contribuye desde una perspectiva teórica académica sobre la importancia del empleo en economía del país, mediante el análisis de incentivos normativos laborales por el impacto de la pandemia en el Ecuador.

\section{Metodologia}

En este apartado de la investigación corresponde examinar algunos aportes teóricos sobre el aspecto laboral y el emprendimiento, para llevar a cabo el estudio se recurrió al paradigma de investigación positivista, tipo de investigación basado en un diseño de campo no experimental y transeccional o transversal con apoyo documental. A decir de, Tamayo (2003) en la investigación de campo los datos se recogen directamente de la realidad, razón por lo que se les denomina primarios, lo cual, según el precitado autor su valor radica en que permite cerciorarse de las verdaderas condiciones en que se han obtenido los datos, lo cual facilita su revisión o modificación en caso de surgir dudas (p.65).

El nivel de investigación fue la investigación descriptiva, atendiendo lo manifestado por Arias (2016) "La investigación descriptiva consiste en la caracterización de un hecho, fenómeno, individuo o grupo, con el fin de establecer su estructura o comportamiento" (p.24).

Corresponde, por tanto, con el nivel descriptivo en función del objetivo general planteado, la población estuvo estructurada por tres sectores seleccionados de microempresas, considerando la base del catastro del SRI del 2021, así Azuay con 45.814, Cañar 10.497 y Morona Santiago 11.300, total 67.611 microempresas de la Zona 6 del Austro, de las cuales son empresas registradas en la Superintendencia de Compañías por el año 2021 en Azuay 8.843, Cañar 494 y Morona Santiago 341, total 9.678, dando un gran total de 77.289, se determinó el tamaño de la muestra con un 4,50\% de error, estableciendo la muestra $n=471$, a su vez, la validez de contenido fue realizado mediante el juicio de tres expertos con experiencia en el área de laboral, contabilidad y metodología.

Por su parte, fue importante analizar en primer lugar, la pertinencia del coeficiente de consistencia interna de Cronbach, el mismo que trata sobre el grado en que los ítems miden la homogeneidad. Para determinar la confiabilidad del cuestionario elaborado, se procedió a aplicar una prueba piloto a 30 empresas, que no forman parte de la muestra, todos ellos con características similares a los sujetos de este estudio, administrando una escala, de 7 ítems, a una muestra de $\mathrm{n}=30$. De acuerdo con los resultados anteriores, se concluyó que la escala tiene una confiabilidad de consistencia interna alta, suficientemente alta como para ser considerada fiable. En conclusión, la confiabilidad del instrumento fue de 0,9 muy alta, así mismo, fue sustancial la utilización de la herramienta estadística para el desarrollo de la investigación, recurriéndose a la estadística descriptiva, lo que permitió la recolección, organización, análisis y presentación de datos con el apoyo de cuadros, tablas, figuras y los resultados obtenidos de la aplicación de los cuestionarios 
en relación con los objetivos planteados del presente estudio, recurriéndose a la estadística descriptiva mediante el software libre SPSS, versión 26.

El análisis de los datos es una parte esencial de toda investigación, en tal sentido, Hernández et al. (2016), sostienen que "el procedimiento que se sigue para analizar los datos consiste en: Una vez que los datos se han codificado, transferido a una matriz, guardado en un archivo y "limpiado" los errores, el investigador procede a analizarlos utilizando un programa computacional" (p. 272). En base a lo cual se procedió a inspeccionar, limpiar y transformar datos con el objetivo de resaltar información útil, lo que sugiere conclusiones, y apoyo a la toma de decisiones. Posterior a los procedimientos que condujeron a reunir datos con el propósito específico de estructurar un análisis de la normativa financiera, se procedió a revisar los datos o respuestas obtenidas mediante Google forms. Las respuestas a los cuestionarios se recopilaron de forma automática y ordenada en formularios, con figuras y datos de las respuestas en tiempo real, que fueron procesados, analizados e interpretados, considerando el uso de la triangulación, validez, confiabilidad y contrastación de los instrumentos.

\section{Fundamentación Teórica}

El marco teórico referencial comprende aspectos teóricos, conceptuales, legales, situacionales de la realidad que forman parte de la presente investigación, garantizando de esta manera la integración e interrelación de la teoría con la metodología y objetivos de investigación.

El impacto en los mercados laborales de cada país de la región depende, entre otras cosas, de la estructura productiva y la composición del empleo de cada sector. Entre las áreas que han sido gravemente afectadas se encuentran las relacionadas con el turismo, aerolíneas, alojamiento, restaurantes y hoteles, el comercio y la industria manufacturera, así como las actividades inmobiliarias y administrativas. Estos sectores son intensivos en mano de obra y algunos, como es el caso del comercio, concentran una elevada proporción de empleo informal. Otros sectores que también soportan un impacto medioalto son el transporte y almacenamiento y las actividades de entretenimiento y culturales. Los trabajadores de estos sectores fueron afectados con particular intensidad por el riesgo económico que deviene del confinamiento y el cambio en las prioridades del consumidor.

La llegada del COVID-19 a los países de América Latina ha redundado en una paralización de la actividad económica, planteando desafíos referentes tanto a la contención de la pandemia como al inicio de una fase de reapertura económica escalonada. Es previsible que la recuperación económica no se produzca en forma generalizada, sino progresivamente en distintos sectores de actividad, así como en ciertas localidades y regiones, siempre bajo las condiciones decretadas por las autoridades sanitarias.

Asimismo, la reapertura de ciertas actividades probablemente no significará la recuperación inmediata de los niveles anteriores a la crisis, sino que se requerirá un tiempo de transición. Varios países de la región hicieron uso de instrumentos que ya 
existían o implementaron nuevas políticas para apoyar a las empresas afectadas con acceso a liquidez y haciéndose cargo parcialmente de los salarios de sus trabajadores, en algunos casos a través de prestaciones de los seguros de desempleo, en otros a través de subsidios directos. El propósito de estas políticas es entregar ingresos a los trabajadores, mantener en pie a las empresas y dar continuidad a la relación laboral con sus trabajadores de forma que se mantengan los colaboradores o trabajadores cuando comience la reactivación económica, este es el desafío prioritario de cara a una recuperación que se producirá en forma gradual en ciertas actividades (OIT, 2020).

En la actual crisis causada por el COVID-19, varios países que cuentan con un seguro de desempleo han ofrecido modalidades de disminución de las horas trabajadas y de las remuneraciones que serán pagadas por el empleador, con una compensación de los ingresos, como es el caso del seguro de cesantía parcial en Alemania, Austria y Suiza. Frente a la crisis actual, estos países han ampliado la población que puede beneficiarse de esas medidas, incorporando, por ejemplo, a trabajadores con contratos a plazo fijo. En la presente coyuntura, hasta finales de abril de 2020, 751.000 empresas alemanas ya anunciaron que se van a acoger a esta modalidad y se espera que más de 5 millones de asalariados se beneficien del subsidio, cifra que equivale a más de tres veces la registrada durante la crisis de 2009 (CEPAL, 2020).

América Latina, varios países están implementando también medidas que buscan fomentar la continuidad de la relación de trabajo en casos de jornada reducida o suspensión temporal, así como asegurar que los trabajadores que sean desvinculados tengan acceso a un mínimo de ingresos mientras se abran las oportunidades para la búsqueda de un nuevo empleo. En los países que ya contaban con seguro de desempleo contributivo, se adaptaron las condiciones de acceso para aumentar su cobertura, se mejoró el monto de los beneficios o se amplió el período durante el cual se puede recibir el subsidio, y se están inyectando recursos fiscales para complementar los fondos acumulados. También existe la posibilidad de brindar oportunidades de actualización o recalificación a quienes se encuentran recibiendo seguro de desempleo, o de ofrecer capacitación en el marco de programas de empleo de emergencia o en proyectos intensivos en mano de obra (OIT, 2020).

En el caso de los trabajadores informales, trabajadoras domésticas y migrantes, las herramientas de políticas públicas son mucho más limitadas, dado que estos carecen de un contrato de trabajo, no están afiliados ni cotizan a la seguridad social y, sobre todo, no tienen acceso a los seguros de desempleo que existen en algunos países. Son dificultades que encuentran también las trabajadoras domésticas remuneradas, en su mayoría informales: menos del 30\% tienen cobertura de la seguridad social en la región; el desafío es especialmente mayor para los trabajadores migrantes, quienes en muchos casos tienen dificultades para acceder a los beneficios y servicios provistos por las políticas públicas (OIT, 2020).

A decir de Cuadrado et al. (2017), "el crecimiento de los pueblos involucra el aporte del Estado, la academia y la empresa, enfatizando el fortalecimiento al sector que 
agrupa a microempresarios o de trabajadores autónomos". (p. 5), Podemos ver entonces que cada negocio está formada según a su función que realiza y apegada a los reglamentos que la ley dispone de igual forma cada una de ellos son entes económicos que generan trabajo y sostenibilidad dentro de la sociedad por más mínimo que sea su aporte en la economía (Becerra, et al., 2020).

La crisis de la COVID-19 también evidencia la vulnerabilidad de las trabajadoras domésticas remuneradas, sobre todo respecto al riesgo de contagio de la enfermedad y la falta de acceso a beneficios sociales. Es llamativa la ausencia de legislación en la región sobre las condiciones de seguridad y salud mínimas de este grupo de personas. La informalidad imperante en el sector implica que, en la práctica, muchas de ellas dejan de recibir ingresos laborales si no pueden asistir a su lugar de trabajo. Además, la aplicación de las normas que permiten mantener el vínculo laboral es mucho más débil en un contexto de crisis, por lo que un gran número de estas trabajadoras deben vivir en los hogares en los que prestan servicio durante la época de cuarentena. Aquellas que son migrantes se encuentran en una situación aún más vulnerable, tanto por las consecuencias de la ruptura del vínculo laboral como al verse forzadas a permanecer en el hogar en el que trabajan (OIT, 2020).

El mercado laboral en tiempos de crisis sanitaria es preocupante, debido a la disminución de empleo y cambios normativos, la crisis sanitaria y las medidas adoptadas para su mitigación han tenido como consecuencia la disminución de plazas de empleo, la modificación de las jornadas laborales y el aumento del empleo informal. Según, el Banco Interamericano de Desarrollo -BID (2020), Ecuador podría perder entre el 4,3\% y 14,4\% de los empleos formales, dependiendo de la duración y severidad de la crisis.

Por su parte, el impacto laboral en tiempos de la crisis sanitaria dentro del Ecuador es aun altamente incierto. Algunas estimaciones señalan que el empleo formal podría caer entre un $-4.3 \%$ y un $-14.4 \%$ en Ecuador en 2020, dependiendo de la intensidad y la duración de la crisis, (BID, 2020). Ciertamente, el efecto sobre el empleo dependerá no solo de la duración de la crisis (tanto en el ámbito nacional como a nivel internacional) y de las respuestas que se den a ella, sino que también viene determinado por algunas debilidades estructurales del mercado laboral ecuatoriano.

En este sentido, en la medida en que la crisis genere despidos, reducciones de horarios, o deterioro de las condiciones laborales, es de esperar que aumente tanto el desempleo como el subempleo y otras categorías de empleo no adecuado. De hecho, en la historia reciente de Ecuador, los episodios de caída del crecimiento económico han venido asociados con una reducción del empleo adecuado y un aumento del subempleo. Por ejemplo, en el periodo 2014- 2016 de desaceleración económica, el subempleo aumentó y el empleo adecuado fue a la baja, en tanto que, en diciembre de 2019, el empleo adecuado representaba un $38.8 \%$ del total de la población en edad de trabajar (PEA), el subempleo se situó en un $17.8 \%$, y otros empleos no plenos supusieron un $28 \%$ de la PEA, con un $10.9 \%$ de empleo no remunerado y un 3.8\% de desempleo (OCDE, 2020). 
Existen tres características del mercado laboral y de la estructura económica de Ecuador que pueden condicionar el impacto de la crisis sobre el empleo. En primer lugar, el gran número de personas vinculadas con la economía informal, lo que deja en una situación de particular vulnerabilidad laboral a muchos trabajadores. En concreto, los empleos en el sector informal representan un $46.7 \%$ del total, y por tanto se trata, en su mayoría, de empleos que están relacionados con la inestabilidad laboral, los bajos ingresos y la precariedad. Esto es particularmente relevante para aquellos trabajadores dentro de la categoría del subempleo o de "otro empleo no pleno", donde los niveles de informalidad alcanzan el $67.5 \%$ y el $65.5 \%$ respectivamente. En segundo lugar, el impacto de la crisis será mayor en determinados sectores económicos, alrededor del 35\% de empleos se encuentran en sectores donde el impacto de la crisis se manifiesta en mayor proporción, en particular comercio, manufactura, servicios de alojamiento y comida, mientras que un $6.7 \%$ del empleo se encuentra en sectores donde el impacto es medio-alto, en particular el transporte, correo y comunicaciones. En tercer lugar, el predominio en Ecuador de Mipymes, en el tejido productivo puede acentuar el impacto sobre el empleo. Las mismas representan un $60 \%$ del empleo en el país, proporción similar al promedio de América latina y el Caribe- ALC. En la medida en que estas empresas tienen menos capacidad para afrontar posibles pérdidas, una buena parte de sus trabajadores podrían estar enfrentando riesgos de pérdida de empleo o de deterioro de sus condiciones de trabajo (OCDE, 2020).

Según, la Organización Internacional del Trabajo - OIT (2020), la economía informal contribuye al empleo, los ingresos y los medios de vida, y en muchos países de ingreso bajo y de ingreso mediano tiene un papel económico significativo.

Además, es oportuno indicar que el número 2 del artículo 11 de la Constitución de la República del Ecuador, prescribe: “Todas las personas son iguales y gozarán de los mismos derechos, deberes y oportunidades. Nadie podrá ser discriminado por razones de etnia, lugar de nacimiento, edad, sexo, identidad de género, identidad cultural, estado civil, idioma, religión, ideología, filiación política, pasado judicial, condición socioeconómica, condición migratoria, orientación sexual, estado de salud, portar VIH, discapacidad, diferencia física; ni por cualquier otra distinción, personal o colectiva, temporal o permanente, que tenga por objeto o resultado menoscabar o anular el reconocimiento, goce o ejercicio de los derechos". La ley sancionará toda forma de discriminación". Postulado que se fortalece mediante la emisión del "Protocolo de prevención y atención de casos de discriminación, acoso laboral y toda forma de violencia contra la mujer en los espacios de trabajo" (Pozo, 2020, p. 5).

El trabajo, en su sentido más amplio, es una manifestación de la capacidad creadora del hombre, en cuya virtud éste transforma las cosas y les confiere un valor. Con el trabajo, el hombre busca un fin y trata de satisfacer sus necesidades, siendo un tema que ha sido ordenado por el Derecho, a través de la historia (Chávez, 1986).

Un contrato individual de trabajo es una relación personalizada, es decir individual, entre un individuo que entrega su fuerza de trabajo a otra u otras a cambio del pago de una determinada cantidad de dinero. La denominación de individual del contrato se refiere a 
la posición del trabajador en la relación laboral (Chávez, 1986), de allí que el contrato individual de trabajo es el convenio en virtud del cual una persona se compromete para con otra u otras a prestar sus servicios lícitos y personales bajo su dependencia, por una remuneración fijada; aspectos que quedan establecidos en un contrato de trabajo.

Acorde al artículo 284 de la Constitución de la República del Ecuador (2008), que señala, uno de los objetivos de la política económica consiste en mantener la estabilidad económica, entendida ésta como el máximo nivel de producción y empleo sostenibles en el tiempo.

El Código de Trabajo (2013) establece las normas relativas al trabajo contenidas en las leyes especiales o en convenios internacionales ratificados por el Ecuador, determinando que serán aplicadas en los casos específicos a las que ellas se refieran, al respecto el Art. 2.- Obligatoriedad del trabajo, el trabajo es un derecho y un deber social. Por su parte Art. 9.- Concepto de trabajador, la persona que se obliga a la prestación del servicio o a la ejecución de la obra se denomina trabajador y puede ser empleado u obrero, igualmente el Art. 10.- Concepto de empleador, La persona o entidad, de cualquier clase que fuere, por cuenta u orden de la cual se ejecuta la obra o a quien se presta el servicio, se denomina empresario o empleador. (p. 3)

Según, la Ley Orgánica de Apoyo Humanitario (2020), para combatir la crisis sanitaria derivada del Covid-19, institucionalizada y aprobada a través de la Asamblea Nacional, según el Art. 1.- Objeto. - (...) establecer medidas de apoyo humanitario, necesarias para enfrentar las consecuencias derivadas de la crisis sanitaria ocasionada por el COVID-19, a través de medidas tendientes a mitigar sus efectos adversos dentro del territorio ecuatoriano; que permitan fomentar la reactivación económica y productiva del Ecuador, con especial énfasis en el ser humano, la contención y reactivación de las economías familiares, empresariales, la popular y solidaria, y en el mantenimiento de las condiciones de empleo.

Por su parte, Sornoza Parrales et al. (2018) menciona que "El desempleo es un problema serio, especialmente en el contexto de las economías en desarrollo como la ecuatoriana". (p. 21)

Así mismo, Sumba Bustamante et al. (2020), manifiesta que el desempleo conlleva una disminución de los ingresos $\mathrm{y}$, por tanto, produce cambios en el estilo de vida. Se producen cambios radicales en la forma de vivir, pues se vive con la incertidumbre de no saber cuánto tiempo durará esta situación. En tal circunstancia, se tiende a ser precavido y reducir drásticamente los gastos. (p. 15)

\section{Resultados}

Para el procesamiento y análisis de la información proporcionada por las micro, pequeñas y medianas empresas, en el presente trabajo de investigación, fue necesario acudir a la técnica de la encuesta y el instrumento del cuestionario, en un total de 30 ítems, seleccionando únicamente cinco por ser los más notables. 
Figura 1

Modalidades de trabajo implementadas por la presencia del COVID-19.

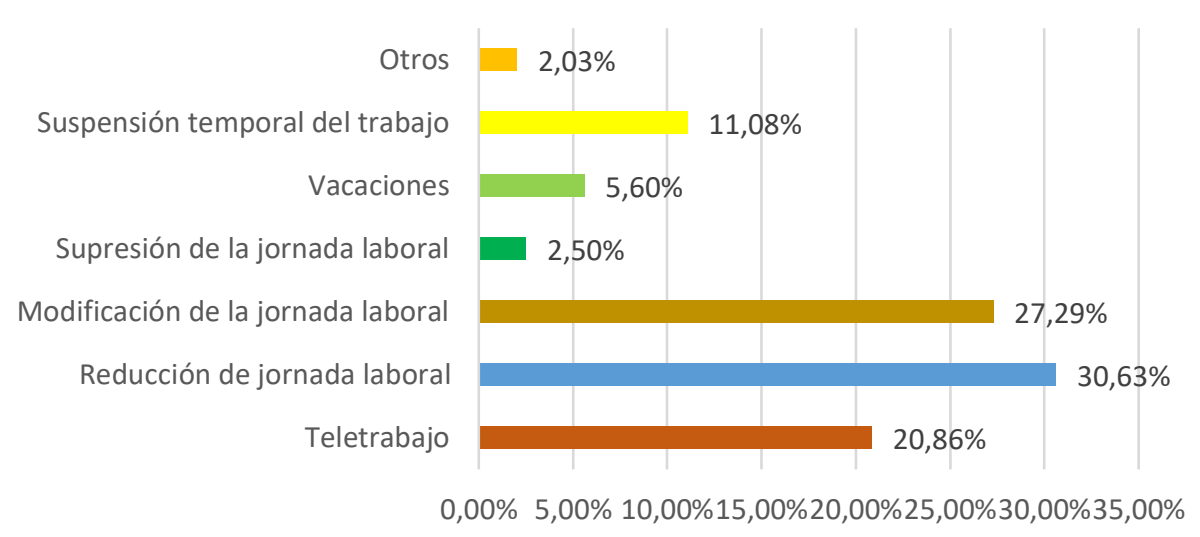

Fuente: Elaboración propia (2021). Tomado del proyecto La importancia de la información contable y financiera ante la incertidumbre del COVID-19 en las Mipymes en el entorno de los negocios en la región 6 .

La información recogida permitió determinar que las principales acciones tomadas ante la crisis económica derivada del Covid-19 fueron: con un 30.63\% la reducción de jornada laboral, seguido de un $27.29 \%$ quienes han modificado la jornada laboral, el $20.86 \%$ optaron por teletrabajo, un $11.08 \%$ suspendieron temporal sus actividades, el $5.06 \%$ proporcionaron vacaciones, el $2.50 \%$ suprimieron la jornada laboral, el $2.03 \%$ asumieron otro tipo de actividades a razón de la emergencia sanitaria. La reducción de jornada laboral sin duda ha beneficiado al empleador en la medida que fue efectiva la disminución de pago de sueldos, así como la modalidad de teletrabajo que consiste en el desempeño de actividades remuneraras o prestación de servicios utilizando como soporte las tecnologías de información y comunicación para el contacto entre el trabajador y la empresa, sin requerirse la presencia física del trabajador en el sitio específico de trabajo; y la modificación de la jornada laboral, situaciones que se han tenido que sobrellevar y que en ocasiones han resultado una alternativa para los empleadores, pero que de cierta manera han perjudicado a los trabajadores quienes percibían un sueldo y que a la final del día terminan desarrollando el mismo trabajo por una remuneración o pago menor.

Figura 1

Razones de despido de trabajadores por la presencia del COVID-19.

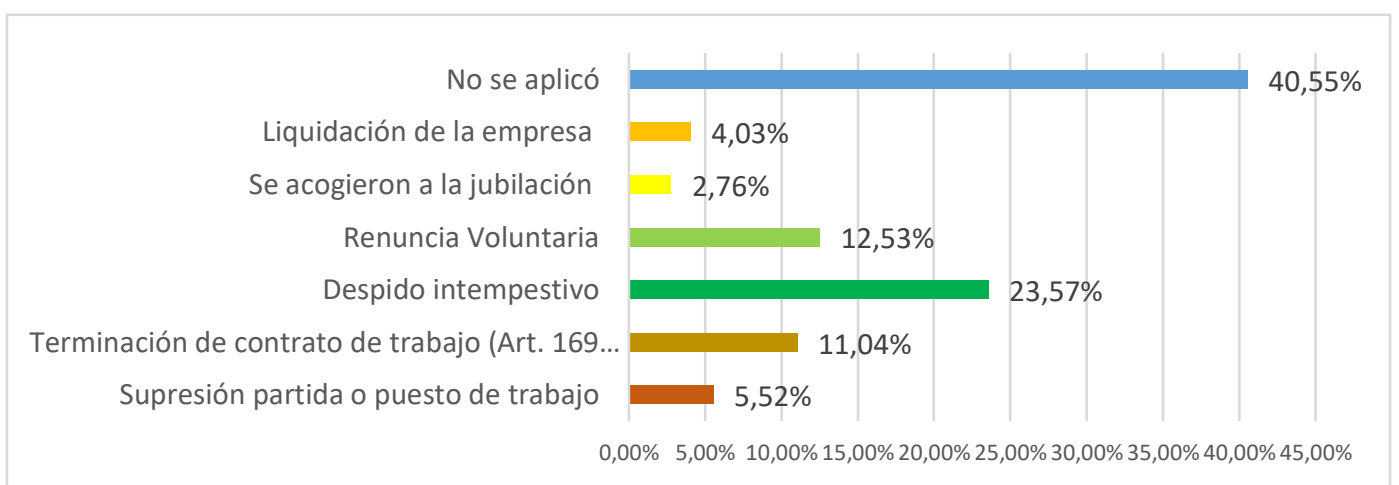

Fuente: Elaboración propia (2021). Tomado del proyecto La importancia de la información contable y financiera ante la incertidumbre del COVID-19 en las Mipymes en el entorno de los negocios en la región 6 . 
El análisis sobre las razones por las que se despidieron a los trabajadores indicó que, un $40.55 \%$ no tuvo que acudir a esta medida, es decir mantuvieron trabajando a sus colaboradores en cualesquiera de las modalidades precedentes en el análisis anterior, sin embargo, dentro de las principales causas por las que existió disminución de personal fueron: se despidió personal intempestivamente en un $23.57 \%$, un $12.53 \%$ renunció voluntariamente, el $11.04 \%$ fue por terminación de contrato de trabajo, el 5.52\% fue por la supresión del puesto de trabajo, el $4.03 \%$ fue a causa de liquidación o cierre de la empresa, el $2.76 \%$ se acogieron a la jubilación. Es posible evidenciar que existe un alto porcentaje de personas que cesaron sus funciones a causa de la supresión de sus actividades, estas medidas fueron asumidas por los dueños de las Mipymes a causa de la temporalidad de pandemia que resultó en grandes pérdidas y bajas en la venta de sus productos y servicios, inclusive en la suspensión temporal de sus actividades, lo cual desencadeno un sinfín de despidos utilizando las amplias posibilidades establecidas en la normativa legal vigente.

\section{Figura 2}

Modalidad de contratado de trabajadores en la Mipymes.

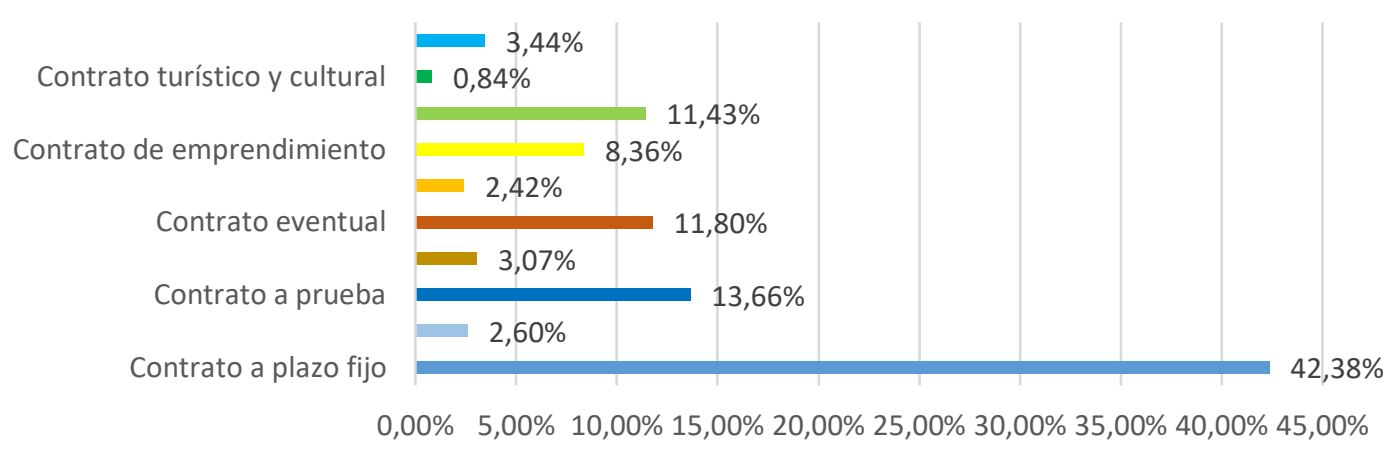

Fuente: Elaboración propia (2021). Tomado del proyecto La importancia de la información contable y financiera ante la incertidumbre del COVID-19 en las Mipymes en el entorno de los negocios en la región 6 .

Con relación a este aspecto, según la figura $\mathrm{N}^{\circ} 3$, el contrato a plazo fijo es la modalidad más usada con un $42.38 \%$, seguido de un $13.66 \%$ de quienes realizan contratos a prueba, el $11.80 \%$ tiene un contrato eventual, el $11.43 \%$ realiza contrato joven, el $8.36 \%$ elabora contratos de emprendimiento, el 3.44\% asume otro tipo de estrategias para contratar a personal, el $2.60 \%$ realiza contratos indefinidos, el $2.42 \%$ realiza contrato productivo, el $0.84 \%$ elabora contratos de tipo turísticos y culturales. La modalidad de plazo fijo estriba en aquel contrato laboral suscrito de manera escrita entre un empleador y trabajador por un determinado tiempo, y los demás contratos como el indefinido, a prueba, obra cierta y eventual, las empresas adquieran compromisos en cuanto a seguro social y demás beneficios de ley que deben ser cumplidas, al estar contratados, así mismo se observa uniendo tendencias entre los contratos de emprendimiento, joven, productivo y turístico y cultural representan 23,05\%, que el Ministerio del Trabajo, con el fin de regularizar y generar más plazas de empleos formales y adecuar las normas contractuales a las distintas actividades económicas y productivas de país, emitió estas cuatro nuevas modalidades y que están creciendo paulatinamente, ello conlleva a una recuperación del empleo, con un 
incremento de ocupación de la población económicamente activa y una reducción de las tasas de desempleo y subempleo.

\section{Figura 3}

Importancia de las nuevas normativas laborales, ante la incertidumbre del COVID-19.

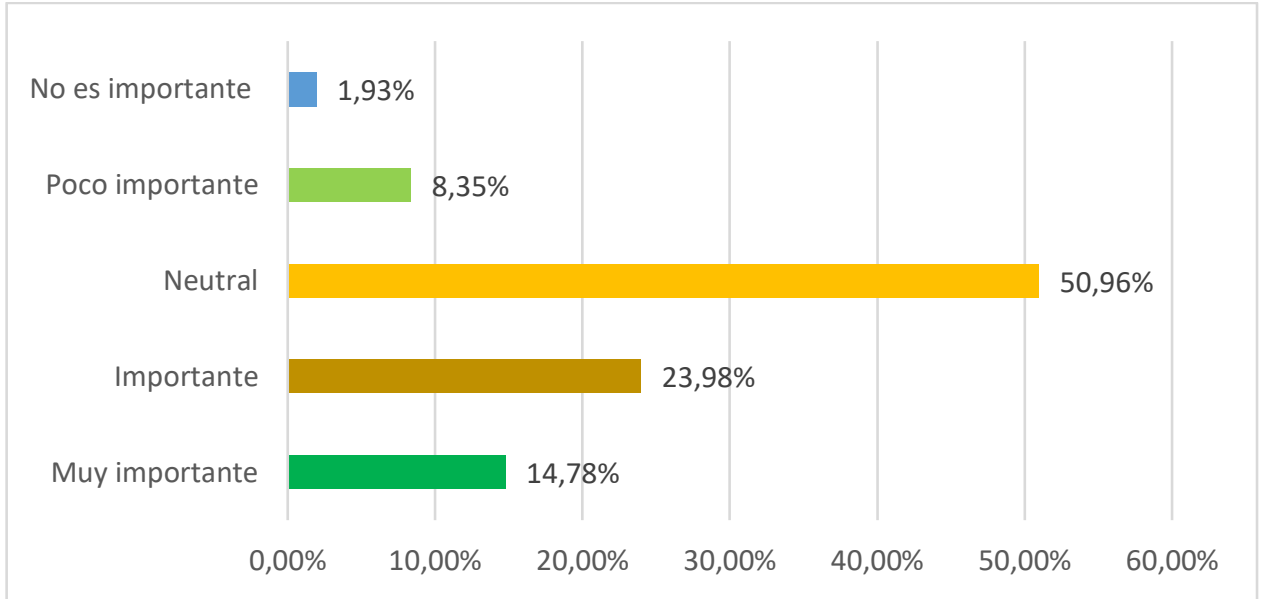

Fuente: Elaboración propia (2021). Tomado del proyecto La importancia de la información contable y financiera ante la incertidumbre del COVID-19 en las Mipymes en el entorno de los negocios en la región 6 .

Como puede advertirse, las nuevas resoluciones y normativa laborales impuestas por el Ministerio del Trabajo han sido tomadas de manera neutral en un 50.96\% del total de la población de las micro, pequeñas y medianas empresas encuestadas, es decir no tienen un criterio que emitir, mientras que el $23.98 \%$ las asumen como importantes, el $14.78 \%$ como muy importantes, uniendo estas tendencias tenemos el $38.76 \%$, como poco importante el $8,35 \%$ y el $1.93 \%$ manifiestan no ser importantes. Al respecto, la Ley Orgánica Humanitaria y las resoluciones emitidas por el Ministerio del Trabajo, permitieron hacer frente a la actual situación económica y sanitaria del país, la Asamblea Nacional aprobó la Ley Orgánica de Apoyo Humanitario, con medidas que permiten fomentar la reactivación económica y productiva del Ecuador y, regula entre otros aspectos: acuerdos laborales, pensiones educativas, tarifas para servicios básicos, facilidades financieras, convenios entre deudores y acreedores e incentivos tributarios para las nuevas inversiones, teniendo como condición fundamental la generación de empleo (Becerra et al., 2021).

Así mismo con el fin de regular el registro de modalidades y acuerdos laborales, además de la aplicación de la reducción emergente de la jornada de trabajo, se expidieron los Acuerdos Ministeriales $\mathrm{N}^{\circ}$ MDT-2020-132 y 133, con fecha 15 de julio de 2020 respectivamente, lo cual a la vista de la difícil situación económica del país para ciertas empresas resulta bastante complicado aplicar, es evidente que al tener un porcentaje mayoritario de quienes se mantienen en un criterio neutral, estas regulaciones pueden ser o no conocidas por los encuestados, quienes llevan el proceso de contratación y el ámbito laboral, aspecto que debería ser difundido a miras de contar con mayor liquidez, sostenibilidad y el desarrollo de sus empresas. 
Figura 4

Opciones aplicadas por las empresas ante la reducción de la jornada, conforme la Ley Orgánica de Apoyo Humanitario.

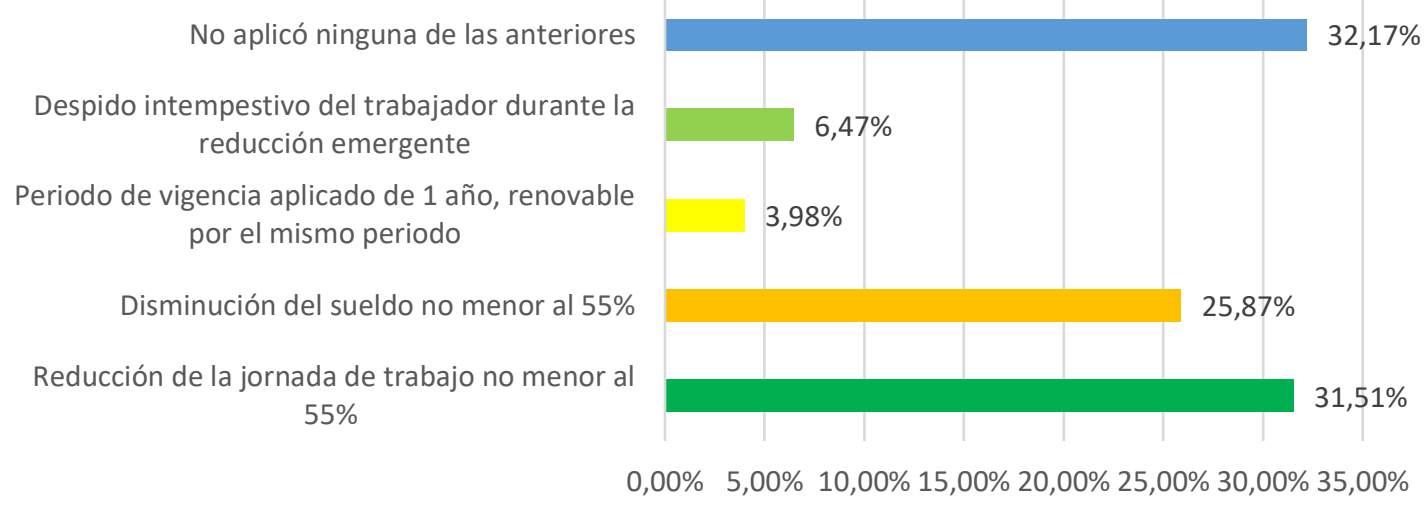

Fuente: Elaboración propia (2021). Tomado del proyecto La importancia de la información contable y financiera ante la incertidumbre del COVID-19 en las Mipymes en el entorno de los negocios en la región 6 .

Los datos contenidos en el grafico $\mathrm{N}^{\circ} 5$, sostienen que el $32.17 \%$ no aplicó ninguna de las opciones que se dieron para la reducción emergente de la jornada, de acuerdo a la ley Orgánica de Apoyo Humanitario, el $31.51 \%$ aplicó la reducción a la jornada de trabajo no menor al $55 \%$, mientras que el $25.87 \%$ optó por la disminución del sueldo no menor al 55\%, para el $6.47 \%$ fue adecuado acogerse al despido intempestivo del trabajador durante la reducción emergente y finalmente el $3.98 \%$ decidió estimar un periodo de vigencia del contrato de trabajo de 1 año, renovable por el mismo periodo. Las diferentes acciones aplicadas han podido ajustarse a las necesidades de las empresas, en donde las condiciones económicas laborales se han visto modificadas, beneficiando a las personas o empresas que perdieron la capacidad de cumplir con los pagos requeridos. Como es lógico, Ley Orgánica de Apoyo Humanitario determinó acuerdos con el fin de lograr la preservación de fuentes de trabajo y garantizar estabilidad a los trabajadores, en este sentido, los trabajadores y empleadores podrán, de común acuerdo, modificar las condiciones económicas de la relación laboral.

\section{Análisis de medidas laborales en las Mipymes}

La presencia del coronavirus en Ecuador no sólo está afectando la salud de sus habitantes, sino en varios contornos de la humanidad como son la educación, salud, transporte público, seguridad, trabajo, el comercio, entre otros.

Todas las empresas, con independencia, sin importar su tamaño, están afrontando graves problemas, como es la disminución de ingresos; aumento de la insolvencia; y, pérdida de puestos de trabajo. Las mipymes serían una de las más perjudicadas dentro del sector empresarial para su sostenimiento y vigencia en el mercado local y nacional. Como es natural el Covid-19 genera actualmente incertidumbre y pánico, no sólo por ser una calamidad pública que ha alterado gravemente el tejido empresarial, económico, social o ecológico de nuestro país; sino también en el ámbito del trabajo, en este contexto es 
importante conocer ¿qué pasará con la cantidad de empleo, que condiciones dieron lugar a desempleo, así como subempleo, surgiendo la incertidumbre sobre la calidad del trabajo, que implicó cierre de empresas, reducción de horas laborables, por tanto disminución de salarios; y el acceso a protección social, que implica diversas políticas y acciones en contornos que deben promover el ejercicio de los derechos económicos, sociales y culturales, en el mercado laboral, la salud, el medio ambiente, la alimentación, el cuidado, entre otros.

Es conveniente destacar que mediante diálogo tripartito entre gobierno, trabajadores y empleadores, se logró ciertos niveles dignos de ingresos, ahora también nace la indecisión e inseguridad con respecto a qué grupos de personas serán los más afectados para conseguir o mantener plazas de trabajo, siendo los más vulnerables el sector informal, los migrantes y las trabajadoras domésticas, de allí que el Estado está dando salida en primer lugar inoculando a la población contra la pandemia, hasta llegar a la curva de inmunidad de rebaño, esto está permitiendo la reactivación de la economía que está muy venida a menos ante el asecho de una tercera ola de la pandemia.

Entre las principales ventajas que proporcionó la Ley Orgánica Humanitaria (2020), fueron enmarcadas en una reforma laboral, en la cual la Comisión de Desarrollo Económico propuso que se haga una interpretación al artículo 169 del Código de Trabajo con el que se pueden dar despidos intempestivos si las empresas argumentan calamidad.

Por su parte, el Ministerio del Trabajo, sobre la aplicación de la Ley Orgánica de Apoyo Humanitario, a través del Acuerdo Ministerial No. MDT-2020-172, respecto del registro de modalidades y acuerdos laborales, permitió evitar que más empleos se pierdan, la transición para regresar al empleo adecuado y ayudó a generar nuevas plazas de trabajo.

La Ley Orgánica de Apoyo Humanitario (2020), en su Art. 10.- Créditos productivos para la reactivación económica y protección del empleo en el sector privado. - A partir de la promulgación de la presente Ley, y con la finalidad de evitar la ruptura de la cadena de pagos, reactivar la economía y proteger el empleo, las entidades del sistema financiero nacional, ofrecerán líneas de crédito al sector productivo, de rápido desembolso que incluirán condiciones especiales, tales como: periodos de gracia, plazos de pago y tasas de interés preferenciales. Becerra y Cuadrado (2020) sostienen que el microcrédito juega un papel esencial como dinamizador de la economía, especialmente en los sectores que tradicionalmente no han sido atendidos por la banca formal.

Conforme, Art. 16.- de la Ley Orgánica de Apoyo Humanitario (2020), relacionados con los acuerdos de preservación de fuentes de trabajo, los trabajadores y empleadores podrán, de común acuerdo, modificar las condiciones económicas de la relación laboral con la finalidad de preservar las fuentes de trabajo y garantizar estabilidad a los trabajadores. Los acuerdos no podrán afectar el salario básico o los salarios sectoriales determinados para jornada completa o su proporcionalidad en caso de jornadas reducidas. El acuerdo podrá ser propuesto tanto por trabajadores como por empleadores. Los empleadores deberán presentar, de forma clara y completa, los sustentos de la necesidad de suscribirlos, para que el trabajador pueda tomar una decisión informada. Una vez 
suscritos los acuerdos, estos deberán ser conocidos por el Ministerio del Trabajo, quien supervisará su cumplimiento. El acuerdo será bilateral y directo entre cada trabajador y el empleador. El acuerdo alcanzado, durante el tiempo de su vigencia, tendrá preferencia sobre cualquier otro acuerdo o contrato. Igualmente, de producirse el despido del trabajador al que se aplica el acuerdo, dentro del primer año de vigencia de esta Ley, las indemnizaciones correspondientes se calcularán con la última remuneración percibida por el trabajador antes del acuerdo.

En torno a la reducción emergente de jornada de trabajo, por eventos de fuerza mayor o caso fortuito debidamente justificados, los empleadores podrán reducir la jornada laboral hasta un máximo del 50\%. El sueldo o salario corresponderá en proporción a las horas efectivamente trabajadas y no será menor al $55 \%$ de la remuneración fijada previo a la reducción. El aporte a la seguridad social y beneficios de ley se pagará en base a la jornada reducida, esta reducción podrá aplicarse hasta por 1 año renovable por el mismo período, por una sola vez.

En cuanto a las empresas que hayan implementado la reducción de la jornada laboral no podrán disminuir el capital social de la empresa ni repartir dividendos obtenidos en los ejercicios en que vigente esta jornada. No se podrá aplicar este régimen por quienes se acogieron a lo establecido en el art. 4 del Acuerdo MDT-2020-080, de 15 de marzo de 2020.

Conforme al contrato especial emergente, se creó un contrato especial con jornadas que pueden ir de 20 a 40 horas semanales, que podrán ser distribuidas en 6 días. Los acuerdos y el nuevo contrato podrán durar 1 año a partir del registro en el Sistema Único de Trabajo - SUT, con la opción de renovarlo por 1 año más. El contrato deberá celebrarse por escrito y registrarse en el SUT dentro de los 15 días contados, a partir de la suscripción del mismo. El pago de la remuneración del contrato emergente se podrá realizar en cualquiera de estas formas: diaria, semanal, quincenal o mensual.

Tal como lo señala la Ley Orgánica Humanitaria, con respecto a la reducción de jornada laboral y acuerdos, los empleadores y trabajadores podrán modificar las condiciones económicas de la relación laboral. La jornada de trabajo podrá ser reducida hasta el $50 \%$ del horario y hasta el 55\% de la remuneración establecido previa a la jornada emergente, el acuerdo entrará en vigencia a partir del registro en el SUT.

En relación al teletrabajo, se acordó en el contrato de trabajo o un documento anexo, la modalidad de teletrabajo en sus diferentes formas: autónomos, móviles, parciales, y ocasionales, es importante señalar que los trabajadores que prestan servicios de teletrabajo gozarán de todos los derechos.

Igualmente, el Art. 22.- Prestaciones del seguro de desempleo, de la Ley Orgánica de Apoyo Humanitario (2020), durante los meses de abril, mayo, junio y julio del año 2020, los afiliados del Instituto Ecuatoriano de Seguridad Social, en relación de dependencia, que pasaren a situación de desempleo, podrán acceder a la prestación del seguro de desempleo. 
Así mismo, de acuerdo al Art. 23.- Requisitos, de la Ley Orgánica de Apoyo Humanitario (2020), la persona afiliada para acceder a la prestación de seguro de desempleo, conforme lo señala el artículo anterior, deberá cumplir los siguientes requisitos: a) Acreditar 24 aportaciones acumuladas y no simultáneas en relación de dependencia, de las cuales al menos 6 deberán ser continuas e inmediatamente anteriores a la contingencia; b) Encontrarse en situación de desempleo por un período no menor a diez (10) días; c) Realizar la solicitud para el pago de la prestación a partir del día octavo de encontrarse desempleado, y hasta en un plazo máximo de 45 días posteriores al plazo establecido en este literal; d) No ser jubilado; $y$,

e) Debe verificarse previamente el aviso de salida registrado por el Empleador en el IESS. Durante los meses de abril, mayo y junio del año 2020, por efecto de la pandemia del COVID 19, los pagos correspondientes al seguro de desempleo se efectuarán automáticamente y sin más trámites, de forma mensual, a partir de que la calificación efectuada por el IESS sea procedente.

Según, El Ministerio del Trabajo del Ecuador (2020), sostuvo que los resultados han sido buenos, 75 mil empleos se han salvado, más de 230 mil empleos se crearon desde la aprobación de la Ley Humanitaria y 25 mil con la aplicación del contrato emergente. Además, se crearon 16.929 nuevos contratos, desde el 30 de junio de 2020.

Según los datos revelados por el Ministro del Trabajo (2020), las nuevas modalidades de contratos por giro de negocio, con la aprobación de la Ley Humanitaria, se activaron en el sector productivo, turismo, emprendimiento, contrato joven y de formación. Aseguró que las modalidades contractuales actuales generan estabilidad para el trabajador, prevén una remuneración justa y beneficios sociales.

Así mismo, con respecto a la regulación del socio trabajador, sobre la aplicación del Acuerdo Interministerial No. MDT-IEPS-2020-001, en el cual se expide la normativa para regular la relación entre la organización asociativa de Economía Popular y Solidaria y el socio trabajador, el Ministro señaló que lo que se trata es de ayudar a identificar la real naturaleza de los socios. La regulación debe ser aplicada para casos específicos, al respecto representantes de la Coalición Nacional de Economía Popular y Solidaria, el acuerdo es ilegítimo porque se los considera como trabajadores cuando son socios, atenta a los principios de economía popular y solidaria, por lo que pidió su derogatoria.

Así mismo, lo que tienen que ver con el reconocimiento de Artesanos, en cuanto a los títulos artesanales que se encuentran refrendados solo por el Ministerio del Trabajo, los títulos deben ser reconocidos, según la Ley de Defensa del Artesano, también por el Ministerio de Educación, según los criterios jurídicos, se reitera que la Junta Nacional de la Defensa del Artesano y el Ministerio del Trabajo constituyen los entes encargados para establecer la formación, titulación, refrendación y calificación artesana, a nivel nacional, garantizando los derechos profesionales y socioeconómicos de los artesanos, de tal manera el Ministerio de Educación se abstiene de participar en dichos procesos. 
Lo que tiene que ver con la renuncia obligatoria, en respuesta a la aplicación de la sentencia de la Corte Constitucional, respecto de la declaratoria de inconstitucionalidad del Decreto $\mathrm{N}^{\circ} 813$ relacionado a la compra de renuncias obligatorias para los servidoras y servidores públicos, se derivó que los efectos son a futuro y no retroactivos, ninguna autoridad va a poder aplicar el decreto que fue declarado inconstitucional, por lo tanto, en ningún caso, se aplicará la compra de renuncias obligatorias y tampoco se puede exigir la devolución de los valores para regresar a la función pública.

\section{Conclusiones}

A continuación, se exponen las principales conclusiones, integrando los resultados de los cuestionarios, y la técnica de la observación:

- La información recogida en los gráficos $\mathrm{N}^{\circ} 1,2$ y 5 respectivamente, sostiene que en los meses más fuertes de la llegada de la pandemia hubo reducción, modificación, suspensión, supresión de la jornada laboral, vacaciones y teletrabajo sumadas las tenencias tenemos el 97,96\%, en tanto que el 40,55\%, no aplicaron ningún despido de trabajadores por la presencia de COVID-19, pero el resto de ejecutivos empresariales, si optaron por despido intempestivo, supresión de partida, terminación de contrato, renuncia voluntaria, jubilación y liquidación por cierre de empresa, sumado esta tendencia constituye el 59,45\%, en tanto que el 31,51\%, aplicaron la reducción de la jornada de trabajo no menor al 55\%, conforme la Ley Orgánica de Apoyo Humanitario y el 32,17\% no aplicó ninguna opción que permitía la citada ley, de allí que, cada función en la relación contractual fluctúa con base a parámetros definidos que permiten crecimiento económico sustentable y sostenible; atender las condiciones del empleo menos remunerado es de igual relevancia para el desarrollo equitativo que el mayormente remunerado, ambos coadyuvan para la generación de tributos, riqueza y desarrollo del país.

- El análisis de los gráficos $\mathrm{N}^{\circ} 3$ y 4 con respecto a la modalidad de contratos que disponen las micro, pequeñas y medianas empresas, el $42,38 \%$ tienen contratos a plazo fijo, que es aquel contrato laboral suscrito de manera escrita entre un empleador y trabajador por un determinado tiempo, que permite que ambas partes comprendan claramente sus obligaciones y las condiciones de empleo. En tanto que los nuevos contratos joven, emprendimiento, productivo, turístico y cultural tienen una tendencia del 23,05\%, estas nuevas modalidades laborales están enfocadas en generar estabilidad para el trabajador durante su vigencia, además se podrán aplicar exclusivamente para nuevas contrataciones y renovarse por una única ocasión, por otra parte, uniendo la tendencia el 38,76\% entre importante y muy importante, con respecto a normativas laborales, el 50,96\% se han mantenido neutral, la Ley Orgánica Humanitaria, planteó una serie de medidas solidarias de alivio social para llevar mejor la cuarentena y evitar posibles despidos de trabajadores.

- Ante la marcada divergencia entre todos los sectores que componen la fuerza laboral y los incentivos otorgados, así como las tenciones que se generaron entre 
trabajadores y empleados, mediante la Ley Orgánica Humanitaria, como una estrategia válida por parte del gobierno a fin de atenuar la curva vertiginosa que compuso el desempleo, se buscó evitar que la informalidad se apodere del mercado laboral, no obstante, existen factores que dificultan que el empleado común pueda ejecutar acciones que lo coloquen con un empleo fijo y un sueldo digno. En Ecuador es difícil cuantificar la informalidad a un número exacto, y a su vez no existen reglamentos específicos que mitiguen o impulsen el crecimiento o decrecimiento del empleo informal.

- Es conveniente destacar, que para superar la pandemia del COVID-19, fue fundamental la actuación rápida de las autoridades gubernamentales, en pro de implementar políticas públicas en el ámbito laboral, las cuales sentaron bases para una recuperación paulatina a todos los niveles de la economía ecuatoriana, apuntando a la protección de los trabajadores en el lugar de trabajo, fomentando la actividad económica y la demanda de mano de obra, será necesario que el gobierno del Presidente Guillermo Laso, apoye al empleo y al mantenimiento de ingresos mediante el incentivo a las inversiones y el consumo, es decir políticas públicas que permitan mitigar los efectos de esta pandemia que tanto daño a causado al tejido productivo, empresarial y al trabajo, protegiendo el recurso humano y garantizando los derechos de los trabajadores y empleadores mutuamente.

- El COVID-19 como agente directamente invasivo, sumado a la salubridad menguada del Ecuador, obligó a proponer al Estado medidas emergentes y desesperadas; y en términos generales, a la adecuación de estándares laborales, los cuales provocaron deprimir las pocas plazas de trabajo que quedaron después de catorce años de gobierno. En el plano numérico, el desempleo se elevó hasta lograr picos estadísticos que no figuraban, las medidas para resarcir dicha afrenta laboral no llegan a suplir el encarecimiento de la vida en épocas de pandemia.

- Los datos recogidos, determinaron que el desempleo absorbió a un gran número de la Población Económicamente Activa- PEA, es la mayor afectada por el coronavirus, trayendo consigo impactos sociales y económicos, de allí que el empleo como principal inyección, tanto emocional como herramienta del poder adquisitivo, es importante en términos micro y macroeconómicos, a la vez que es imperativo para salvaguardar los intereses de la población sacudida por un virus extraño, cuyo impacto se sale de las manos de las autoridades gubernamentales.

\section{Referencias bibliográficas}

Arias, F. (2016). El Proyecto de Investigación: Introducción a la metodología científica. (7 $7^{a}$ Edición), Caracas - Venezuela. Editorial El pasillo, C.A.

Constitución de la República de Ecuador (2008). (2008, 20 de octubre). Asamblea

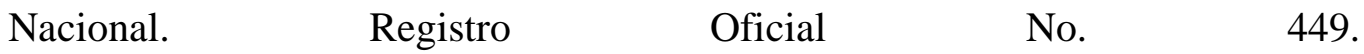
http://www.estade.org/legislacion/normativa/leyes/constitucion2008.pdf 
Banco Interamericano de Desarrollo (BID), 2020. (Abril de 2020). ¿Cómo impactará la COVID-19 al empleo?,

en. https://publications.iadb.org/publications/spanish/document/C\%C3\%B3 mo_impactar\%C3\%A1_la_COVID-

19_al_empleo_Posibles_escenarios_para_Am\%C3\%A9rica_Latina_y_el_ Caribe.pdf

Becerra, E. y Cuadrado, G. (2020). El microcrédito productivo como dinamizador de la economía campesina en la región del Austro. Visionario Digital,4(4), 6-31. https://www.cienciadigital.org/revistacienciadigital2/index.php/VisionarioDigita 1/article/view/1416

Becerra, E. Flores, M. y Cuadrado, G. (2021). La fiscalidad en las Mipymes de la región del Austro, en el contexto de la pandemia del COVID19. AlfaPublicaciones, 3(3),

98-119. https://www.alfapublicaciones.com/index.php/alfapublicaciones/article/view/71

Becerra, E., Jaramillo, Y., \& Astudillo, P. (2021). Alcance de la información societaria, durante la pandemia del COVID-19. Alpha publicaciones, 3(3), 120-138. https://doi.org/10.33262/ap.v3i3.72

Chávez, J. (1986). Contrato individual del contrato. (2021, 10 de agosto) Ecuador: https://biblio.flacsoandes.edu.ec/catalog/resGet.php?resId=44530.

Cuadrado, C., Macas, Á., Calle, O. y Vintimilla, S. (2017). La universidad, los programas de capacitación y las asociaciones de comercio informal. RECIMUNDO: Revista Científica de la Investigación y el Conocimiento, 1(5), 254-270.

El Código de Trabajo (2013). Codificación 17,p ublicada en el Registro Oficial Suplemento 167 de 16-Dic-2005 contiene hasta la reforma del 26-Sep-2012

El Ministerio del Trabajo del Ecuador (2020), (julio de 2021). Aplicación de ley humanitaria en el ámbito Laboral. https://www.asambleanacional.gob.ec/es/noticia/69576- ministro-de-trabajoinformo-sobre-aplicacion-de-ley

Hernández, S., Fernández, C., \& Baptista, L. (2016). Metodología de la Investigación. México: Mc Graw Hill Educación, 6ta. Edición.

La Comisión Económica para América Latina y el Caribe- CEPAL. (Abril de 2020). Dimensionar los efectos del COVID-19 para pensar en la reactivación. https://www.cepal.org/es/publicaciones/45445-dimensionar-efectos-covid-19pensar-la-reactivacion.

Ley Orgánica de apoyo humanitario (2020). Gaceta oficial $N^{\circ} 229$, del 22 de junio del 2020. 
Organización Internacion del Trabajo- OIT. (Mayo de 2020). Coyuntura Laboral en. Coyuntura Laboral en.

https://www.cepal.org/sites/default/files/publication/files/45557/S2000307_es.

pdf.

Organización para la Cooperación y Desarrollo Económicos- OCDE. (2020). Impacto social del COVID-19 . Impacto social del COVID-19 . https://www.oecd.org/dev/Impacto-social-COVID-19-Ecuador.pdf.

Pozo, H. (22 de diciembre de 2020). Ministerio del Trabajo. registro oficial organo Republica del Ecuador. Quito, Ecuador: https://www.trabajo.gob.ec/wpcontent/uploads/2020/11/2020-Acuerdo-Ministerial-Nro.-MDT2020\%E2\%80\%93244.pdf?x42051.

Sornoza, D. R., Parrales, M. L., Sornoza, G. I., Cañarte, T. C., Castillo, M. A., GuarandaSornoza, V. F. y Delgado, H. B. (2018). Fundamentos de emprendimiento. 3Ciencias. http://dx.doi.org/10.17993/EcoOrgyCso.2018.39

Sumba Bustamante, R. Y., Saltos Ruiz, G. R., Rodríguez Suarez, C. A., y Tumbaco Santiana, Z. L. (2020). El desempleo en el ecuador: causas y consecuencias. Polo del Conocimiento, 5(50), 774-797. http://dx.doi.org/10.23857/pc.v5i10.1851

Tamayo, M. (2003). El Proceso de Investigación Científica (4 edición). México. Editorial Limusa. 


\section{PARA CITAR EL ARTÍCULO INDEXADO.}

Becerra Molina, E., Cuadrado Sánchez, G., \& Astudillo Arias, P. (2021). Consecuencias sobre el empleo por el impacto del COVID-19, en el entorno de los negocios de la Región 6. ConcienciaDigital, 4(4), 85-105. https://doi.org/10.33262/concienciadigital.v4i4.1905

\section{LCiencia}

El artículo que se publica es de exclusiva responsabilidad de los autores y no necesariamente reflejan el pensamiento de la Revista Conciencia Digital.

El artículo queda en propiedad de la revista y, por tanto, su publicación parcial y/o total en otro medio tiene que ser autorizado por el director de la Revista Conciencia Digital.

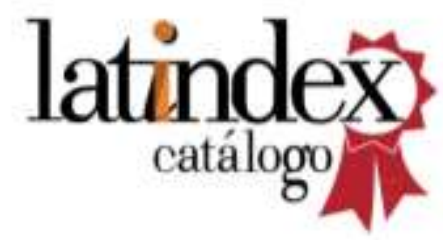

